ICAS $054 / 20$

\title{
Estimating COVID-19 cases and outbreaks on-stream through phone-calls
}

\author{
Ezequiel Alvarez $^{(a) \dagger}$, Daniela Obando ${ }^{(b)}$, Sebastian Crespo ${ }^{(b)}$, Enio Garcia ${ }^{(b)}$, \\ Nicolas Kreplak ${ }^{(b)}$, Franco Marsico ${ }^{(b) \star}$
}

(a) International Center for Advanced Studies (ICAS), ICIFI-CONICET \& ECyT-UNSAM, Campus Miguelete, 25 de Mayo y Francia, CP1650, San Martín, Buenos Aires, Argentina

(b) Ministerio de Salud de la Provincia de Buenos Aires, La Plata, Buenos Aires, Argentina

\begin{abstract}
One of the main problems in controlling COVID-19 epidemic spread is the delay in confirming cases. Having information on changes in the epidemic evolution or outbreaks rise before lab-confirmation is crucial in decision making for Public Health policies. We present an algorithm to estimate on-stream the number of COVID-19 cases using the data from telephone calls to a COVID-line. By modeling the calls as background (proportional to population) plus signal (proportional to infected), we fit the calls in Province of Buenos Aires (Argentina) with coefficient of determination $R^{2}>0.85$. This result allows us to estimate the number of cases given the number of calls from a specific district, days before the lab results are available. We validate the algorithm with real data. We show how to use the algorithm to track on-stream the epidemic, and present the Early Outbreak Alarm to detect outbreaks in advance to lab results. One key point in the developed algorithm is a detailed track of the uncertainties in the estimations, since the alarm uses the significance of the observables as a main indicator to detect an anomaly. We present the details of the explicit example in Villa Azul (Quilmes) where this tool resulted crucial to control an outbreak on time. The presented tools have been designed in urgency with the available data at the time of the development, and therefore have their limitations which we describe and discuss. We consider possible improvements on the tools, many of which are currently under development.
\end{abstract}

E-mail: $\dagger$ sequi@unsam.edu.ar, $\star$ fmarsico@mincyt.gob.ar, 


\section{Introduction}

The COVID-19 epidemic is causing a global damage to practically all aspects of world society since early 2020. Although a huge effort in many fields of sciences is being made to mitigate its effects, the disease is continuously spreading and, in many regions, a second wave is causing great concerns. The difficulties in controlling the epidemic are in part due to a crucial combination of being highly contagious [1], having a long incubation period [2] during which contagions are possible a few days before symptoms onset [3], having mild or asymptomatic cases [1] and also because the diagnosis may take a few days since contacting the Health Care system. In particular, the latter yields that outbreaks spread and epidemic evolves while laboratory results are being processed. This effect being more important in low and medium income countries due to operational and logistic problems, generally caused by technological and economic inequalities $[4,5]$.

We present in this work a method to mitigate the epidemic effects by estimating the number of COVID-19 cases without having to wait for lab confirmations. This provides the Health Care system a tool to react in advance and evaluate current or next Public Health policies.

In mass accidents or major catastrophes Early Warning Systems (EWS) play a key role for disaster mitigation [6-8] decreasing response times and improving their effectiveness. The main strategy of EWS in infectious disease surveillance is the incorporation of information produced nearly from the infection $[9,10]$. In this case, the symptoms onset and their detection by the individual and community health perception is the first detectable signal of cases and, in particular, an outbreak. EWS based on syndromic surveillance have been applied in epidemiological surveillance for early outbreaks identification and confirmation [11-15]. One of the main characteristics of EWS is the utilization of health information provided by the population in order to activate local alarms. Nowadays, with the wide use of cell phone applications and specific health-system phone lines, important databases with information about syndromic surveillance are generated each day [16]. Geo-location plays a main role is spatial and temporal definition of the outbreaks detected by EWS [17].

In Buenos Aires Province (Argentina), the COVID-19 phone line 148 is one of the first contacts between a person that believes to be infected and the Health Care System. The trained Health Care team receives and responds to people questions generating, simultaneously, a syndromic surveillance database. If the person has symptoms that could indicate a COVID19 infection, it is instructed to follow the corresponding protocol. Importantly, such syndromic database was used as input for estimation of cases and outbreak detection in Buenos Aires Province.

This work is divided as follows. In Section 2 we describe the COVID-line data and present the details of the mathematical model to estimate the number of cases using the phone calls data. In Section 3 we show how the model works in Buenos Aires Province and how it can be used to track on-stream the epidemic. In Section 4 we present the Early Outbreak Alarm 
medRxiv preprint doi: https://doi.org/10.1101/2020.10.09.20210351; this version posted October 13, 2020. The copyright holder for this preprint (which was not certified by peer review) is the author/funder, who has granted medRxiv a license to display the preprint in perpetuity.

It is made available under a CC-BY-NC-ND 4.0 International license .

and show its details in Villa Azul (Quilmes) case. We discuss the limitations and current improvements of the model in Section 5, and we present our conclusions in section 6 .

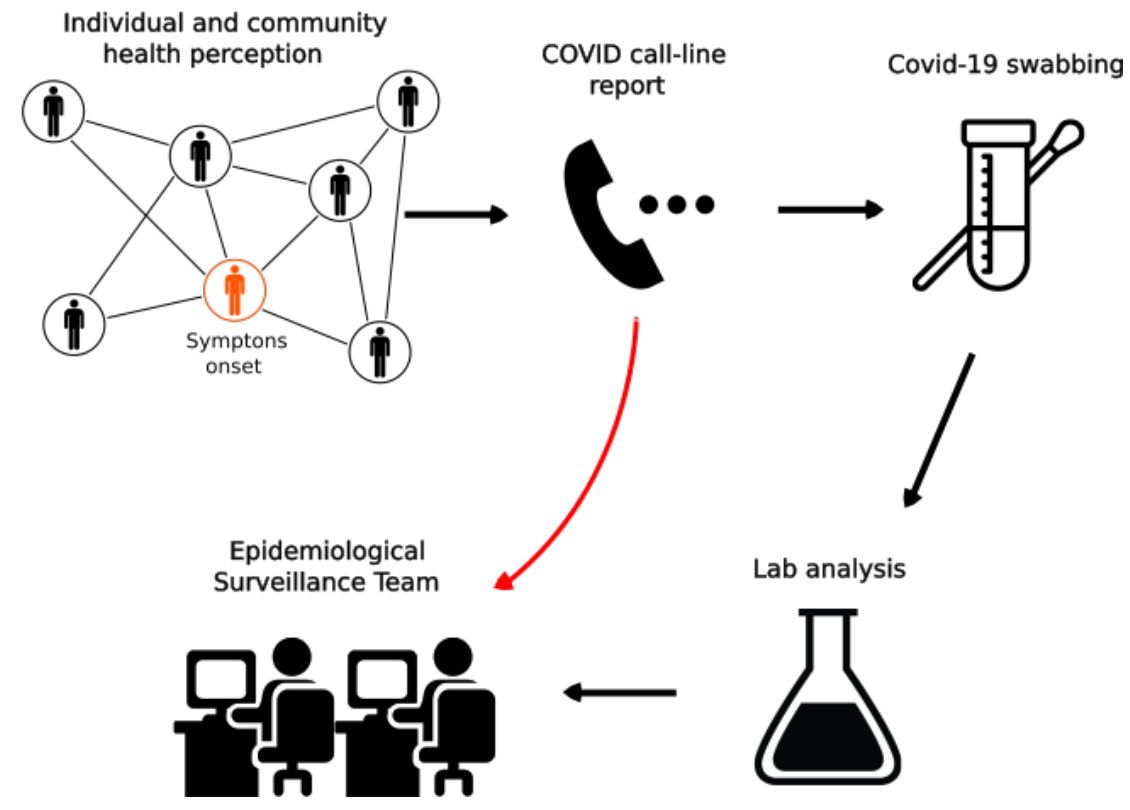

Figure 1: COVID-line 148 workflow. As people call the COVID-line upon their health perception, the COVID-trained operators determine whether they correspond to suspicious or close-contact case. In such a case, their record is passed to the Epidemiological Surveillance team and a COVID-19 swabbing is ordered. Some days later the swabbing lab result is added to the corresponding record. The algorithm described in this paper works with the first part of the information which is delivered on-stream as the operators determine the case passes the corresponding threshold.

\section{Estimating on-stream COVID-19 cases through calls to a COVID-line}

We describe the mathematical model implemented to relate phone calls to a COVID-line to lab-confirmed cases per district per day. In the following paragraphs we outline the functioning of the 148 COVID-line and then we describe the details of the model.

\subsection{COVID-line 148 in Buenos Aires Province}

Buenos Aires Province (PBA for its acronym in Spanish) is the most populated province of Argentina, with more than 17 million inhabitants. Around 13 million people live in the 
medRxiv preprint doi: https://doi.org/10.1101/2020.10.09.20210351; this version posted October 13, 2020. The copyright holder for this preprint (which was not certified by peer review) is the author/funder, who has granted medRxiv a license to display the preprint in perpetuity.

It is made available under a CC-BY-NC-ND 4.0 International license .

Metropolitan area surrounding the City of Buenos Aires. Importantly, the remaining 4 million live in the vast area and with low population density known as the interior of the province. This demographic heterogeneity leads to a hyper-centralized Health Care system. In order to attend the growing demand for medical assistance caused by the COVID-19, public health authorities implemented in February 2020 a specific-COVID phone that is reached by dialing 148. The objective of this COVID-line is to address all community concerns related to COVID, which includes questions, doubts, symptom reports, and reference to the Health Care system, among others.

The COVID-line grew in personal as the epidemic spread in PBA. The call request grew from a few hundreds per day at March up to approximately 20k per day at the end of August. Until late June the system was not outperformed and all calls requiring assistance were taken. We therefore consider that during this regime an indicator coming from this COVID-line would be relatively unbiased. This is specially true if one compares this to other indicators as testing, or lab processing, which were changing their behavior considerably as epidemic spread during this period. We find that April 1st to June 26th is a period in which the COVID-line has been relatively stable to major changes.

As people call the COVID-line 148, they enter into an automatic voice menu in which one of the options corresponds to COVID-like symptoms. As user go into this option their call is taken by a COVID-trained operator and a short questionnaire on their experience indicates whether the call does not pass the threshold to be registered or corresponds to one of the two registered categories: close-contact and suspicious case. If the call corresponds to any of these categories, then the operator registers their data and in particular the district from which they are calling. We depict in Fig. 1 the workflow of the COVID-line. At the early stage that the system was implemented, the record did not include trustful information on the exact address of the user. This crucial fact led us to develop the system we explain below by restricting our information on the user to only their district. Although future upgrades on the system are providing more accurate location of the call, the current work restricts to the caller district and only once their call is taken by a COVID-trained operator.

\subsection{Mathematical Model to estimate cases from phone-calls to the 148 COVID-line}

We present the mathematical model to estimate the new infected using the phone call data, and apply it to PBA. The reasoning in this section follows the same lines as in Ref. [18], but with different purposes and different filtering in the data set.

We consider a data set of calls from many districts and during a given time range to a COVID-line. Each one of these calls can either be

background: people with similar symptoms but not infected

signal: people infected with COVID-19. 
Under reasonable assumptions of homogeneity in space and time we can model that background calls in each district and time-window are proportional to the total district population and the time-window length. Whereas signal calls are proportional to the total number of infected people in the district whose record is opened in the corresponding time-window, even though their lab-confirmation may be available in a later time. Therefore, if we divide all our data set in chunks corresponding in space to the districts in PBA, and in time to timewindows of $\Delta t^{(j)}$ days that can be arbitrarily chosen, we can pose the following equations for all the chunks labeled by $j$ :

$$
n_{c}^{(j)}=\theta_{p} \Delta t^{(j)} N_{p}^{(j)}+\theta_{I} N_{I}^{(j)}
$$

Where $N_{p}^{(j)}$ is the population of the corresponding district and $N_{I}^{(j)}$ is the number of confirmed infected at the same district and whose record was opened during the corresponding timewindow. On the Left Hand Side, $n_{c}^{(j)}$ is the fit to the total number of calls, whereas $N_{c}^{(j)}$ (not in the equation) is the total number of actually placed calls. Observe, therefore, that this set of equations (one for each chunk $j$ ) can be extended depending on the chosen time-window length. Once this set of $j=1 \ldots k$ equations has been posed, we can fit the best values of coefficients $\theta_{p, I}$ that minimize the square distance between $n_{c}^{(j)}$ and $N_{c}^{(j)}$. We stress that there are only two coefficients $\left(\theta_{p, I}\right)$ that must fit all $k$ different equations for each chunk.

This fit works better if all chunks correspond to periods in which the testing methods have not changed drastically, as it can be for instance if the number of daily tests is modified considerably, or if new symptoms are considered as threshold for testing, among others. The reason for requiring this is to have a coherent balance between the number of infected reported and the number of calls in all chunks all the time. With this objective, is better to re-fit the parameters every time there are major changes in the testing and reporting methods.

Once the parameters $\theta_{p, I}$ in Eq. 1 have been fitted, including their uncertainty from the fit, we can estimate the number of new infected in a given chunk as

$$
n_{I}^{(j)}=\frac{1}{\theta_{I}}\left(N_{c}^{(j)}-\theta_{p} \Delta t^{(j)} N_{p}^{(j)}\right) .
$$

Observe that the Right Hand Side requires data that is obtained in the same day, and therefore one can estimate the number of cases $n_{I}$ on-stream, without need of waiting the laboratory results. Notice, that the algorithm allows to estimate the total number of new cases in each chunk, but not to determine which of the calls correspond to the new cases. The uncertainty in the estimation $n_{I}^{(j)}$ is computed by applying the usual error expansion formula on Eq. 2. If variables are correlated, as for instance $\theta_{p}$ and $\theta_{I}$, one should take this into account, however in our case we neglected this correlation in comparison to other terms. For the parameters $\theta_{p, I}$ we use the uncertainty coming from the fit, for $N_{c}$ we use Poisson uncertainty, and for $N_{p}$ one should decide whether to add a systematic uncertainty or only use Poisson, as we did in this work. As discussed below, uncertainties in the estimations play a central role in the design of the Early Outbreak Alarm, and therefore should be handled with care, specially the systematic ones if present. 
In order to apply this algorithm in PBA we have used the data set of phone calls to the 148 COVID-line. We work with all the phone calls entering the COVID-line that reach the threshold for being close contact or suspicious case. The reason for this filtering is because the district the user is calling from is registered by the operator. Although the address is also in principle registered most of the times, in the practice many ambiguities, misspelled words, or other unintended errors yield that only about $\sim 50 \%-70 \%$ of the times it can be correctly reconstructed. We consider the data set of calls between April 1st and June 26th, since after this the call center was overloaded and not all phone could be taken, yielding intractable biases. Along this period we have fitted the data a few times in different data sets, obtaining fairly similar results and with coefficient of determination always satisfying $R^{2}>0.85$. In particular, as cases were increasing, we were obtaining more accurate estimations for $\theta_{I}$, as it can be expected.

In order to show the robustness of the hypotheses, we show how this model works with data from May 1st to June 26th, divided in two equal length time-windows each. We consider all districts in PBA whose number of calls in these chunks is greater than 100. After this filtering we are left with 43 chunks, i.e. 43 data points. After performing the fit indicated in Eq. 1 we obtain

$$
\begin{aligned}
& \theta_{p}=(5.16 \pm 1.59) \times 10^{-6} \text { calls per inhabitant per day } \\
& \theta_{I}=0.69 \pm 0.05 \text { calls per infected people }
\end{aligned}
$$

It is worth noticing that the precise values of these fitted coefficients have a strong dependence on the process of call filtering and call system architecture. In particular, these values differ from those in Ref. [18] because we are considering a different level of filtering to obtain the district of each user. The fit for this data set yields a coefficient of determination $R^{2}=0.91$, which indicates the robustness of the involved hypotheses. In Fig. 2 we show the comparison between data and fit for the number of phone-calls, as posed in Eq. 1.

\section{Tracking epidemic through model estimations}

The mathematical model described in the previous section provides a framework to estimate many days in advance the number of lab-confirmed cases per day, as a function of the spatiotemporal distribution of phone calls to the COVID-line. This is a compelling achievement because the phone call information is available on-stream, whereas lab confirmation of cases may require from a few days to up to a week since patient report their first symptoms. Along this section we show how this system can be used to have an estimate of the epidemic evolution on-stream, along with real case results in PBA.

As this system was developed there was no time for validation. However, obtaining a very satisfactory $R^{2} \gtrsim 0.85$ in the fit was a signal that the model was insofar working well. As months went by, we had the possibility of comparing in a long range time-window the model estimation against the measured lab-confirmed number of cases per day per district. 


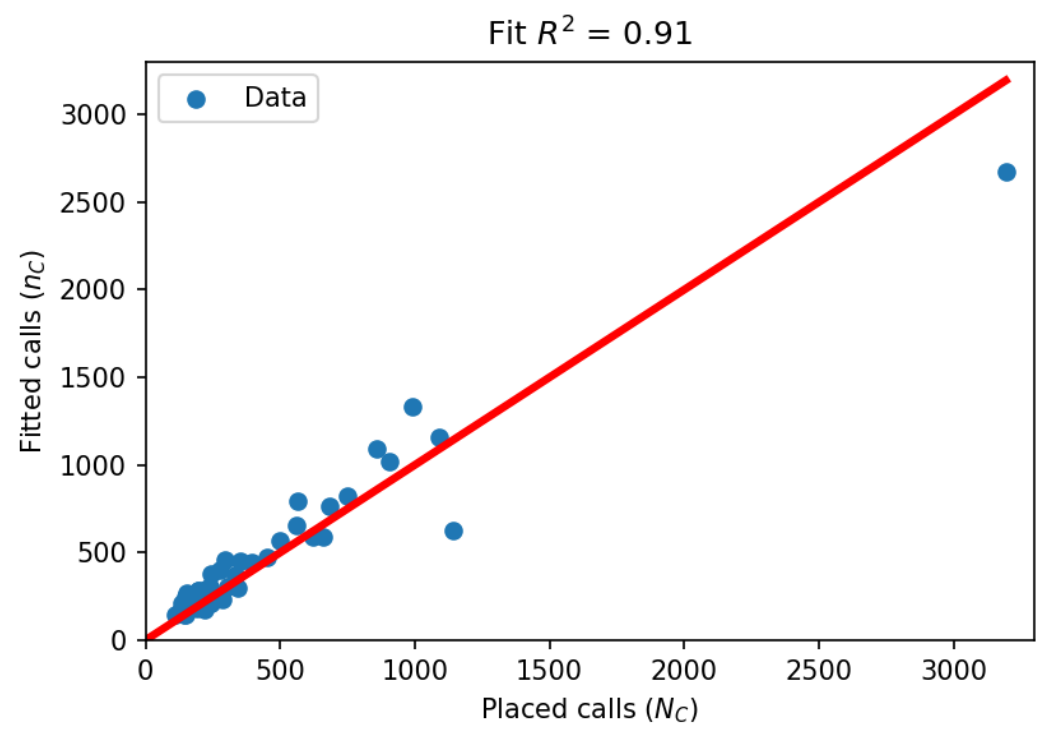

Figure 2: Placed versus fitted calls during the fitted period (May 1st to June 26th divided in two time-windows). The number of fitted calls comes out from the number of laboratoryconfirmed COVID cases using the fit in Eq. 1. The upper right data-point corresponds to La Matanza district whose population of $1.7 \mathrm{M}$ inhabitants is about at least three times larger than all other districts.

In Fig. 3 we show the comparison between the estimation and the late lab-confirmed cases per day for two any districts in PBA. Similar results are obtained for other districts. Is central to observe in this figure that the number of lab-confirmed cases (red line) is an information that is available many days after the corresponding date, whereas the model estimation (blue) is available at the end of each day. As it can be seen in the figure the estimation has a good agreement with the real data. There are a few date ranges in which door-to-door swabbing through DETECTAR operatives [19] induce an expected sub-estimation in cases.

This syndromic surveillance has been used to follow the size, spread, and tempo of outbreaks, to monitor disease trends, and to provide reassurance that potential outbreak has not occurred. In particular, it has also been very useful as an early outbreak detection, as we detail in next section. Syndromic surveillance systems seek to use existing health data in real time to provide immediate analysis and feedback to those in charge with investigation and follow-up of potential outbreaks. Particularly, the data collected by the COVID-line calls proved to be a valuable and reliable input to track the epidemic along the PBA.

The tracking of the epidemic through this model is specially useful when the capacity overload of the diagnostic centers leads to delays in obtaining results. For this reason, having a real-time and relatively unbiased estimation of cases gives the Public Health authorities the possibility of taking actions in time [13]. Furthermore, in a disaster scenario prioritization 
medRxiv preprint doi: https://doi.org/10.1101/2020.10.09.20210351; this version posted October 13, 2020. The copyright holder for this preprint (which was not certified by peer review) is the author/funder, who has granted medRxiv a license to display the preprint in perpetuity.

It is made available under a CC-BY-NC-ND 4.0 International license .

this tool takes a main role when resources and time are limited, as it occurred at the end of June in PBA. The calls-based syndromic surveillance allowed a rapid characterization of the different PBA districts in terms of their epidemiological status and the consequent action was taken in order to mitigate the epidemic effects.
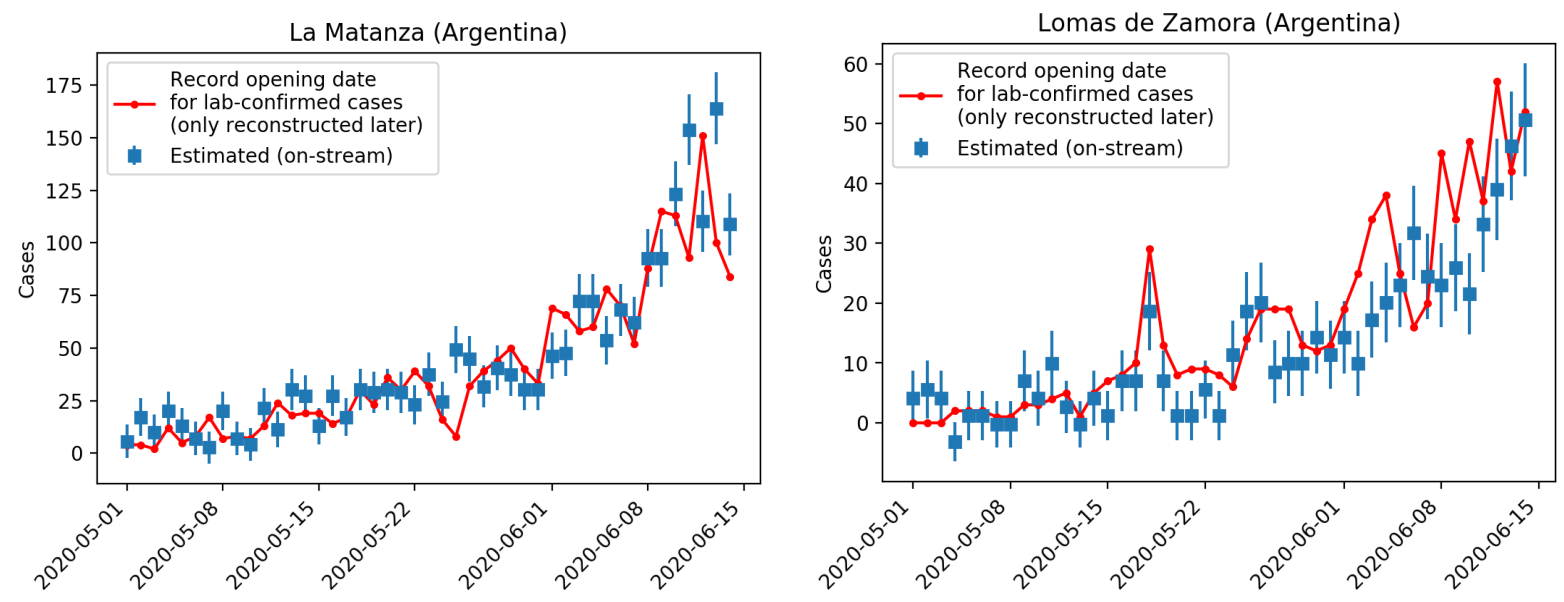

Figure 3: Comparison of real data (red) versus model estimation (blue) for two example districts of Buenos Aires Province (La Matanza and Lomas de Zamora). Recall that the red line corresponding to the real confirmed cases with their record opening in the corresponding date, is reconstructed many days later. In the dates in which the red line goes above the estimation is usually because DETECTAR operatives (door-to-door testing [19]) were carried on. In general, the model yields a very good estimation to monitor the epidemic in all affected PBA districts.

\section{Early Outbreak Alarm}

Along this section we detail a compelling by-product of the model in Section 2 to detect COVID-19 outbreaks considerably earlier than through lab confirmation. We briefly describe the working of the model and then provide its detail through the description of a real case that occurred in mid May in Villa Azul (Quilmes) and Villa Itatí (Avellaneda) in PBA.

\subsection{Identifying an outbreak formation}

Provided the on-stream estimation of the cases per day in each district, we are interested in developing a statistical and automatic tool that can trigger an alarm when a potential outbreak is on rise. Having an early alarm on this kind of epidemiological features is a crucial tool to avoid its spread and drastic consequences. 
To detect a potential outbreak there are many indicators that should be simultaneously analyzed. On one hand it is important to have an estimation of the daily absolute and relative number of cases, and on the other hand is also important to have an estimation on the daily variation of these observables. To have an objective quantitative indicator of the potential of an outbreak in a given region, it is essential to have a correct assessment of the uncertainties in all the estimations of the model. Along the system implemented as an Early Outbreak Alarm, we have considered that the important indicator is the significance of all the basic indicators which signal an anomaly as they depart from zero. Here significance is defined as the distance to zero from the central value of the indicator, measured in units of its uncertainty. Or, in other words,

$$
\text { significance }=\frac{\text { central value }}{\text { uncertainty }} \text {. }
$$

As it can be seen in Eq. 3, the correct computation of the uncertainties (or error bars) is crucial for the functioning of the Early Outbreak Alarm.

The developed algorithm computes everyday the estimation for the total number of new cases in each district in PBA. Since in the studied time-window, specially before June, the number of estimated cases per day of many districts were below $\sim 5-10$, we considered to include the estimation of cases for the last two days. This would reduce the relative Poisson uncertainty due to small numbers. We computed the number of estimated cases in absolute value, and also relative to 100k inhabitants to be equally sensitive to all districts.

A third and decisive observable that signals the level of danger of an outbreak is the daily increase of estimated cases. Given the daily estimation provided by the mathematical model, we can recognize a rapidly increasing curve in many ways. We have considered to fit a straight line to the case estimation for the last three days and use the slope of this line as an estimation of the central value of the daily increase. We also use the significance as the most relevant indicator to decide the level of danger of each district. In this case, the computation of the error bar in the slope of the line includes all the uncertainties in each day estimation included in the computation of the fit uncertainty through the least squares residuals. We use three days to fit a line because is the minimum time needed to see a two-day consecutive increase, while being still well ahead of the laboratory results. In addition, three days is also a good time-window for the specific COVID-19 characteristics.

This Early Outbreak Alarm has provided to PBA Health Care administration very important tools to identify possible outbreaks during the rise of the epidemic curve. Since the granularity of the algorithm is very poor (districts), the system needs to be complemented with other independent indicators, in particular those which can help to provide a more accurate location of the outbreak. This was usually done by calling back manually the recorded cases, and then by sending DETECTAR operatives [19] to verify if in fact the in-situ conditions would be the predicted. The Early Outbreak Alarm has indicated many outbreaks that have been controlled since mid-April to mid-June. In particular, we describe in the following 
medRxiv preprint doi: https://doi.org/10.1101/2020.10.09.20210351; this version posted October 13, 2020. The copyright holder for this preprint (which was not certified by peer review) is the author/funder, who has granted medRxiv a license to display the preprint in perpetuity.

It is made available under a CC-BY-NC-ND 4.0 International license .

paragraphs the very special ${ }^{1}$ case of Villa Azul (Quilmes) and provide the details on how the Early Outbreak Alarm indicated the Quilmes district.

\subsection{Case study: Villa Azul, Quilmes}

We report the details of one of the outbreaks indicated by the Early Outbreak Alarm in midMay in Quilmes district. This case was the first major outbreak in a low-income neighborhood in PBA and had a great impact in the news [20], not only for its magnitude but also because of its early detection that drove a strict lock-down and isolation of the outbreak to control its spread to the close neighborhoods.
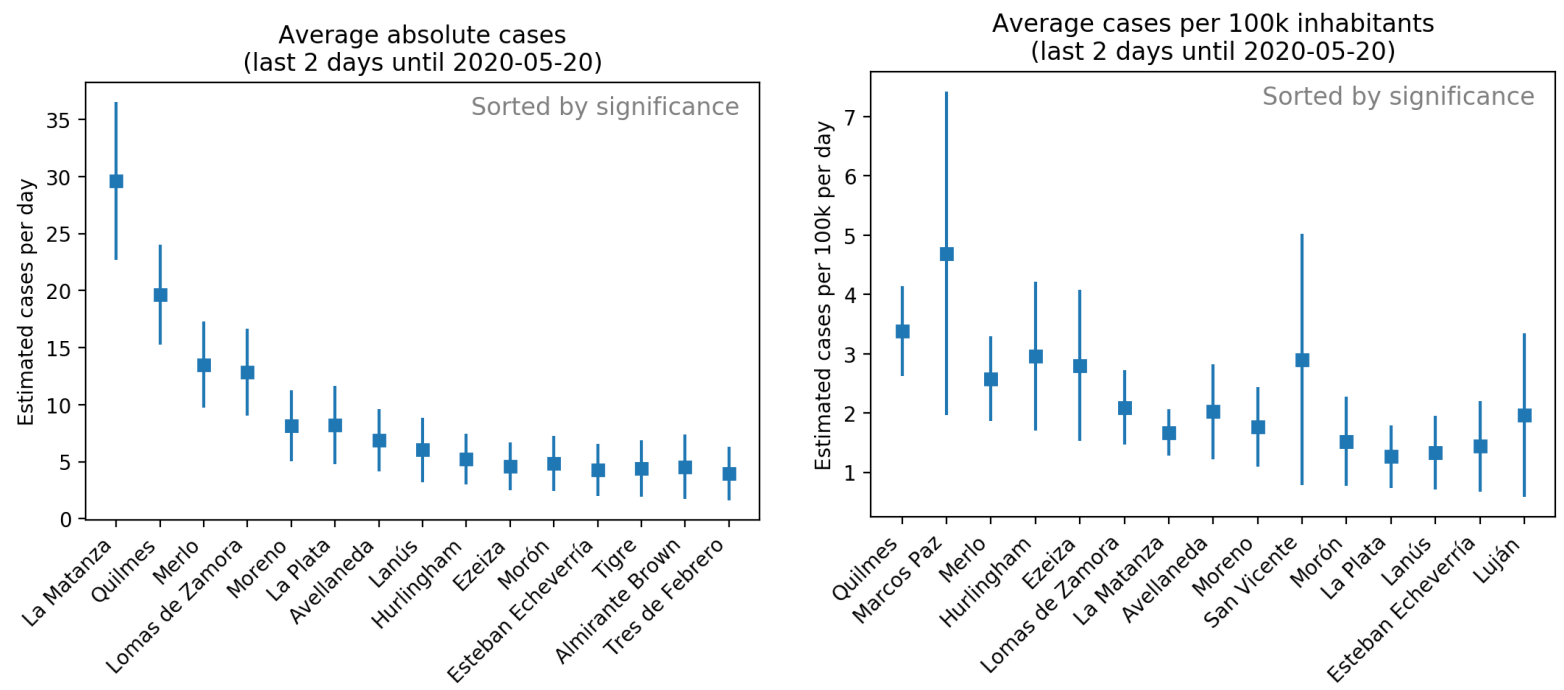

Figure 4: Cases-per-day estimation using the model on the COVID-line phone calls for the last two days. Observe that districts are not sorted by their central value, but by their significance, which is defined as the rate between the central value and the uncertainty. This is why error bars are crucial to provide an Early Outbreak Alarm. Results are shown in absolute value (left) and relative to every 100k inhabitants (right). In the figure we show the scenario for May 20th in which Quilmes is almost as large as La Matanza in absolute value, with $\sim 1 / 3$ of its population. Whereas Quilmes is in the top position when scaled to relative per $100 \mathrm{k}$ inhabitants.

On May 20th the alarm was indicating a large number of estimated cases in Quilmes district (see Fig. 4), in particular Quilmes had the top estimation in number of cases per inhabitants of the last two days, as measured through the significance of the indicator. In addition to this, the indicator of the daily increase fit was also indicating Quilmes as the top district in significance (see Fig. 5). This last indicator on the daily increase fit to the

\footnotetext{
${ }^{1}$ This case covered the headlines in the news for several weeks [20]
} 
Fit to last 3 days

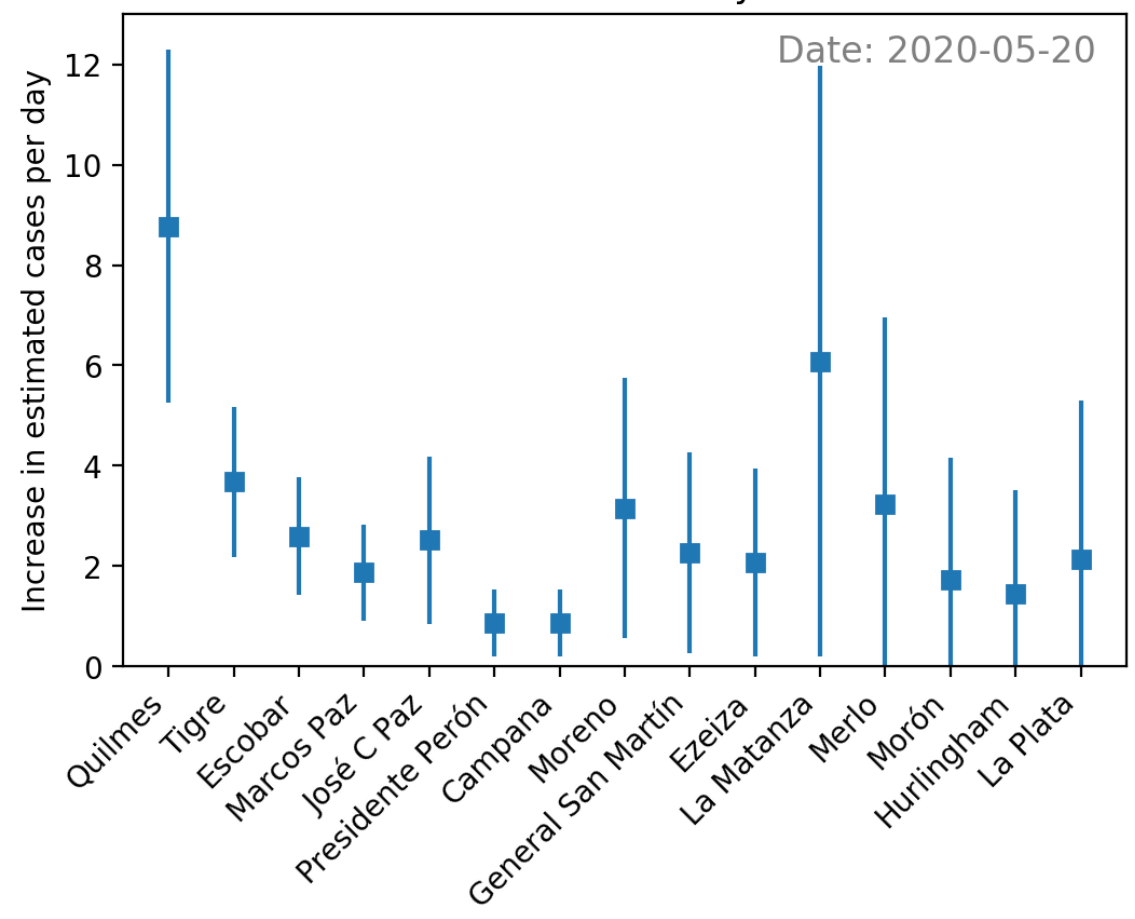

Figure 5: Slope of a linear fit to the cases per day estimation of last three days. The error bar corresponds to including the uncertainty in each per day estimation and in the slope determination in the fit. Also in this plot is crucial to sort the districts according to the significance in this variable. In the figure we see on May 20th Quilmes in the top position, indicating a potential early alarm for an outbreak, as it was consequently confirmed by other indicators a few days later.

last three days can be visualized in Fig. 6a, where we plot the daily estimation for the last 7 days on Quilmes. The fit is obtained using the last three data points in red. Given all these indicators pointing to Quilmes district, the surveillance team took the duty to locate the phone calls and observed an excess coming from Villa Azul, a low-income neighborhood in Quilmes and next to Avellaneda district.

These observations indicated in advance by the Early Outbreak Alarm needed to be verified by an independent complementary indicator. On the following day a DETECTAR operative [19] was sent to Villa Azul, where the acute situation was verified and door-to-door swabbing with urgent lab results started right away. As first results were confirming the outbreak in Villa Azul, the PBA Administration decided a strict lock-down and isolation for 14 days since may 24th [20]. 

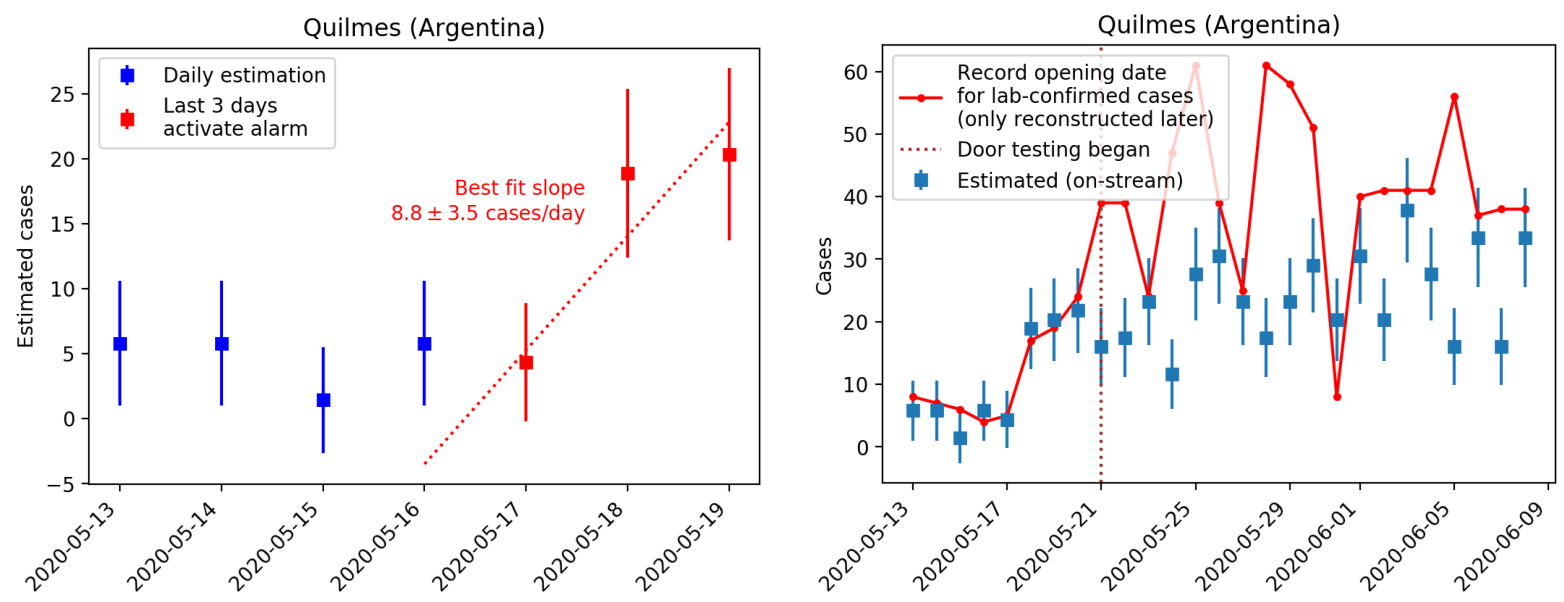

Figure 6: Left: Exact Early Outbreak Alarm visualization on May 20th for Quilmes, as available to the Health Care team in Buenos Aires Province. Right: The posterior picture including the lab-confirmed cases (solid red), the day that the Health Care System landed on Villa Azul to begin testing door by door, and a wider range of dates to capture the big picture of the case. The strict lock-down in Villa Azul with no entering nor leaving permission lasted from May 24th to June 8th. As it can be seen in the plot, during the door-by-door testing, the solid red line goes above and uncorrelated to the estimated cases by phone calls, as expected.

\subsection{Villa Azul epidemiological and operational description}

Villa Azul (Quilmes) and Villa Itatí (Avellaneda) are two adjacent low-income neighborhoods. The last demographic analysis indicates that Villa Azul has a population of 3.128 and Villa Itatí 15.142. High density building and housing, and tiny streets bring the population into close contact. These characteristics make these neighborhoods susceptible to a fast spread [21]. Taking this into account, early outbreak detection implies a main challenge in these complex cases where detection and propagation block must be done when the first few cases are reported. In particular, the above described early alarm for the outbreak occurred in Villa Azul allowing a fast response of the Health Care System team to mitigate and control its propagation to Villa Itatí.

Once produced the strict lock-down and isolation, water and food-supply were delivered by the social care-team. People were not allowed to leave the house during the entire isolation. Active surveillance health teams started with a door-to-door symptoms monitoring. Those cases with clinical manifestation related to COVID-19 were tested. Confirmed cases were isolated inside their houses in cases where this was possible (if there was an empty room for example) and in cases where it was not, the people were sent to an out-of-Hospital center. 
medRxiv preprint doi: https://doi.org/10.1101/2020.10.09.20210351; this version posted October 13, 2020. The copyright holder for this preprint (which was not certified by peer review) is the author/funder, who has granted medRxiv a license to display the preprint in perpetuity.

It is made available under a CC-BY-NC-ND 4.0 International license .

\section{Outlook and scope}

The development of the mathematical model to estimate the number of COVID-19 cases was done in urgency and adapting it to the available data. There was no time to request changes in data acquisition nor processing. Of course the algorithm and the system can be improved in many directions. We discuss some of these features in the following paragraphs.

One of the major weakness in the algorithm is the large granularity, which corresponds to districts. Districts population in the relevant area are in average 500k people. This issue is translated in that the Early Outbreak Alarm stops working once the density of cases is such that there are more than a few outbreaks in each district. This happened in late June in PBA. In a future implementation we are carrying out a workaround for this issue by obtaining a trustful address from the COVID-trained operator that takes de call. A more stable solution would be to obtain this information from the telephone company, however regulations many times block this possibility.

On the other hand, the algorithm has a very important benefit that is its unbiasedness. Given that the COVID-line works 24 hours the 7 days of the week and with a fair equal methodology all the time, the algorithm estimation does not rely on tests availability or overloaded testing facilities, among others. Of course, the system does have slight biases that may come -for instance- from different backgrounds due to different features in the districts, or seasonally social behavior as months go by. Some of these biases may be solved by re-fitting the model once in a while, others by fitting different models in different regions.

Importantly, the algorithm provides information about the background calls that vary in space and time. Further studies could be done in order to understand and extract properties of the background, as it can be its seasonality, variations according to regions, to public announcements or news, etc.

The crucial point in the mathematical model is that recognizes anomalies due to collective behaviors. Therefore, we find that the mathematical model and the Early Outbreak Alarm algorithms can be useful for many other epidemiological diseases - as for instance Dengue-, and other events such as natural catastrophes, among others. We are currently working in the improvement of this system in many aspects, also including Machine Learning algorithms, and these advancements will be published in a future work.

\section{Conclusions}

We have created a syndromic surveillance algorithm based in the correlation between phone calls to a COVID-line, districts population and reported cases. This algorithm works by understanding that phone calls to a COVID-line are a part due to non-infected people having similar symptoms (background) and other part due to infected people (signal). By observing that background has to be proportional to district population, whereas signal proportional to 
reported cases, we have fitted our assumption. The coefficient of determination for Buenos Aires Province (PBA) is always $R^{2}>0.85$ for different samples, which indicates the robustness of our hypothesis. In addition, we have validated our model with real data.

Along the manuscript the model, its estimations, and how we compute their error bars were described. Also, it has been shown how the estimations, which are obtained in-stream, can be used to address Public Health policies without requiring to wait for lab results, which require many more days to converge. The algorithm worked in PBA from April to June, since during this time the COVID-trained call center was not overloaded. Therefore the estimation was relatively unbiased.

We have shown how this estimation can be used to create an Early Outbreak Alarm. Furthermore, we describe how the construction of indicators that have to do with daily cases, and daily increase of cases, can indicate outbreaks in advance. The relevant statistic variable in this case is the significance, since it is a real measure on how far from zero are the indicators. Importantly, this system can detect an outbreak and, in particular, we exemplify its application in the outbreak detection in Villa Azul (Quilmes).

The limitations on the Early Outbreak Alarm were discussed in this paper, many of them because of the characteristics of the data available at the moment of its (urgent) development. We have pointed out many ways to improve its sensitivity and accuracy, on which we are currently working. This alarm would also be useful, not only for other epidemiological diseases, but also for events that yield changes in collective behavior, such as Dengue epidemic, natural catastrophes, or others.

The presented algorithm and mathematical model have been one of the main tools in the PBA Health Care system dashboard during the epidemic, and its current and upgrade versions are still being used to track the epidemic and detect outbreaks.

\section{Acknowledgment}

We thank the fantastic work performed by the 148 COVID call center, in particular to R. Vaena, P. Rispoli and L. H. Molinari for useful conversations. E.A. and F.M. thank Dr. I. Caridi for useful conversations. E.A. thanks CONICET, UNSAM, CAF and EasytechGreen for finantial and logistic support during this research.

\section{References}

[1] Gao, M., Yang, L., Chen, X., Deng, Y., Yang, S., Xu, H., ... and Gao, X. (2020). A study on infectivity of asymptomatic SARS-CoV-2 carriers. Respiratory Medicine, 106026. 
[2] Lee, H., Kim, K., Choi, K., Hong, S., Son, H., and Ryu, S. (2020). Incubation period of the coronavirus disease 2019 (COVID-19) in Busan, South Korea. Journal of Infection and Chemotherapy, 26(9), 1011-1013.

[3] He, X., Lau, E. H., Wu, P., Deng, X., Wang, J., Hao, X., ... and Mo, X. (2020). Temporal dynamics in viral shedding and transmissibility of COVID-19. Nature medicine, 26(5), $672-675$.

[4] Ahmed, O. B. (2020). Mitigating the spread of COVID-19 in low-income countries. Scientific Research and Essays, 15(2), 41-44.

[5] Verhagen, L. M., de Groot, R., Lawrence, C. A., Taljaard, J., Cotton, M. F., and Rabie, H. (2020). COVID-19 response in low-and middle-income countries: Don't overlook the role of mobile phone communication. International Journal of Infectious Diseases, 99, 334-337.

[6] Texier, G., Pellegrin, L., Vignal, C., Meynard, J. B., Deparis, X., and Chaudet, H. (2017). Dealing with uncertainty when using a surveillance system. International Journal of Medical Informatics, 104, 65-73.

[7] Goniewicz, K., and Burkle, F. M. (2019). Disaster early warning systems: the potential role and limitations of emerging text and data messaging mitigation capabilities. Disaster medicine and public health preparedness, 13(4), 709-712.

[8] Kyriacos, U., Jelsma, J., James, M., and Jordan, S. (2014). Monitoring vital signs: development of a modified early warning scoring (MEWS) system for general wards in a developing country. PloS one, 9(1), e87073.

[9] Ginsberg, J., Mohebbi, M. H., Patel, R. S., Brammer, L., Smolinski, M. S., and Brilliant, L. (2009). Detecting influenza epidemics using search engine query data. Nature, 457(7232), 1012-1014.

[10] Krause, G., Altmann, D., Faensen, D., Porten, K., Benzler, J., Pfoch, T., ... and Claus, H. (2007). SurvNet electronic surveillance system for infectious disease outbreaks, Germany. Emerging infectious diseases, 13(10), 1548.

[11] Lombardo, J., Burkom, H., Elbert, E., Magruder, S., Lewis, S. H., Loschen, W., ... and Pavlin, J. (2003). A systems overview of the electronic surveillance system for the early notification of community-based epidemics (ESSENCE II). Journal of urban health, 80(1), i32-i42.

[12] Pavlin, J. A. (2003). Investigation of disease outbreaks detected by "syndromic" surveillance systems. Journal of Urban Health, 80(1), i107-i114.

[13] Katz, R., May, L., Baker, J., and Test, E. (2011). Redefining syndromic surveillance. Journal of epidemiology and global health, 1(1), 21-31. 
[14] Stoto, M. A., Schonlau, M., and Mariano, L. T. (2004). Syndromic surveillance: is it worth the effort?. Chance, 17(1), 19-24.

[15] Hope, K., Durrheim, D. N., d'Espaignet, E. T., and Dalton, C. (2006). Syndromic surveillance: is it a useful tool for local outbreak detection?.

[16] Diwan, V., Agnihotri, D., and Hulth, A. (2015). Collecting syndromic surveillance data by mobile phone in rural India: implementation and feasibility. Global health action, $8(1), 26608$.

[17] Chen, D., Cunningham, J., Moore, K., and Tian, J. (2011). Spatial and temporal aberration detection methods for disease outbreaks in syndromic surveillance systems. Annals of GIS, 17(4), 211-220.

[18] E. Alvarez and F. Marsico, 'COVID-19 mild cases determination from correlating COVID-line calls to reported cases', doi.org/10.1101/2020.07.26.20162008

[19] DETECTAR Operatives site at Argentina.gob.ar/coronavirus/detectar

[20] Impact on major Argentinian newspapers as Clarín (Spanish), BATimes (English) and La Nación (Spanish), among others.

[21] Finch, W. H., and Hernández Finch, M. E. (2020). Poverty and Covid-19: rates of incidence and deaths in the United States during the first 10 weeks of the pandemic. Frontiers in Sociology, 5, 47. 